Chirurg 2019 90 (Suppl 2):S76 https://doi.org/10.1007/s00104-019-0864-x Online publiziert: 13. Februar 2019

(c) Springer Medizin Verlag GmbH, ein Teil von Springer Nature 2019

\section{Originalpublikation}

Sasaki K, Margonis GA, Maitani K et al (2017) The prognostic impact of determining resection margin status for multiple colorectal metastases according to the margin of the largest lesion. Ann Surg Oncol 24:2438-2446

Hintergrund. Die Resektion von Lebermetastasen ist ein prognosebestimmender Bestandteil der multimodalen Therapie des kolorektalen Karzinoms. Bis zu $50 \%$ der resezierten Patienten erleiden dennoch im Verlauf ein intrahepatisches Rezidiv. Während die prognostische Bedeutung einer R1-Situation bei solitären Metastasen gut untersucht ist, besteht Unklarheit über den prognostischen Einfluss positiver Resektionsränder bei multiplen Lebermetastasen.

Methoden. Patienten, die zwischen 2000 und 2015 am Johns Hopkins Hospital eine Resektion von multiplen kolorektalen Lebermetastasen (KRLM) in kurativer Intention erhielten, wurden in die Studie eingeschlossen. Patienten mit einem R1-Status (tumorfreier Resektionsrand $<1 \mathrm{~mm}$ ) für mindestens eine der KRLM wurden in zwei Gruppen aufgeteilt. Der ersten Gruppe wurden Patienten zugeteilt, deren größte KRLM einen R0-Status aufwies und der R1-Status durch eine kleinere KRLM bestimmt wurde („R1 Typ 1“). Der zweiten Gruppe wurden Patienten zugeteilt, deren größte KRLM einen R1-Status aufwies („R1 Typ 2“).

Dieser Beitrag wurde erstpubliziert in Der Chirurg (2017) 88:1060-1061. https://doi.org/ 10.1007/s00104-017-0537-6

M. Schrempf $\cdot$ M. Anthuber

Klinik für Allgemein-, Viszeral- und Transplantationschirurgie, Klinikum Augsburg, Augsburg, Deutschland

\title{
Prognostische Bedeutung der Resektionsränder bei multiplen kolorektalen Lebermetastasen
}

Ergebnisse. Insgesamt 251 Patienten wurden in die Studie eingeschlossen. 156 Patienten hatten negative Tumorränder (R0), 50 Patienten mit R1-Status wurden dem Typ 1 zugeordnet und $45 \mathrm{~Pa}$ tienten mit R1-Status dem Typ 2. Die mediane Nachbeobachtungszeit betrug 30,3 Monate. Das mediane Gesamtüberleben unterschied sich nicht zwischen Patienten mit tumorfreien Resektionsrändern (R0) und Patienten der Gruppe R1 Typ 1 (66,3 vs. 53,3 Monate; $p=$ 0,66). Es zeigte sich jedoch ein deutlich schlechteres medianes Gesamtüberleben für Patienten der Gruppe R1 Typ 2 und zwar sowohl im Vergleich mit Patienten der R0-Gruppe (36,5 vs. 66,6 Monate; $p=0,002)$ als auch im Vergleich mit Patienten der Gruppe R1 Typ 1 (36,5 vs. 53,3 Monate; $p=0,03)$. In der multivariaten Analyse war der alleinige R1Status nach Korrektur für klinische und pathologische Risikofaktoren nicht mit einer schlechteren Prognose im Vergleich zum R0-Status assoziiert (Hazard Ratio [HR] 1,56; $95 \%$-Konfidenzintervall [KI] $0,95-2,57 ; p=0,08)$. Wurde jedoch die Metastasengröße beachtet und lag der R1-Befund an der größten KRLM vor (R1 Typ 2), so war der R1-Status mit einer deutlich schlechteren Prognose in der multivariaten Analyse assoziiert (HR 1,93; $95 \%$-KI 1,04-3,57; $p=0,03$ ).

Diskussion und Fazit. In der hier vorgestellten Arbeit zeigte sich, dass ein positiver Resektionsrand (R1) bei Patienten mit multiplen KRLM dann einen Einfluss auf die Prognose nimmt, wenn er an der größten Lebermetastase vorliegt. Ekberg et al. etablierten in den 1980er-Jahren die heute obsolete 1-cm-Regel in der Leber- metastasenchirurgie [1]. Aktuellere Arbeiten aus der Ära der modernen Chemotherapie zeigten, dass tumorfreie Resektionsränder im Millimeter- und Submillimeterbereich keine Assoziation mit einem schlechteren Gesamtüberleben aufweisen und stellten die prognostische Bedeutung eines mikroskopisch positiven Resektionsrandes sogar vollständig infrage [2]. Die Ergebnisse der hier vorgestellten Arbeit zeigen, dass beim Vorliegen multipler KRLM der Metastasengröße eine entscheidende Bedeutung zukommen könnte. Sollten zukünftige Arbeiten diese Ergebnisse bestätigen, könnte dies zu einer neuen Definition und Bewertung des R1-Status führen.

\section{Korrespondenzadresse}

\section{Prof. Dr. M. Anthuber}

Klinik für Allgemein-, Viszeral- und Transplantationschirurgie, Klinikum Augsburg Stenglinstr. 2, 86156 Augsburg, Deutschland matthias.anthuber@klinikum-augsburg.de

Interessenkonflikt. M. Schrempf und M. Anthuber geben an, dass kein Interessenkonflikt besteht.

\section{Literatur}

1. Ekberg H, Tranberg KG, Andersson R et al (1986) Determinants of survival in liver resection for colorectal secondaries. Br J Surg 73:727-731

2. de Haas RJ, Wicherts DA, Flores E et al (2008) $\mathrm{R} 1$ resection by necessity for colorectal liver metastases: is it still a contraindication to surgery? Ann Surg 248:626-637 